\title{
Tracking Ebola through cellphone, internet of things and Blockchain technology
}

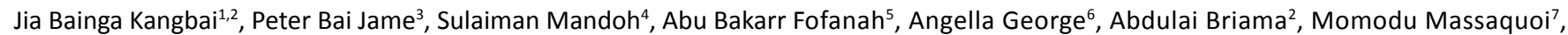
John L McBrayer ${ }^{8}$

Kangbai JB, Jame PB, Mandoh S, et al. Tracking Ebola through cellphone, internet of things and blockchain technology. Curr Res Integr Med 2018; 1(2): $19-21$.

Objective: In the absence of any approved therapeutics and vaccines to treat or prevent Ebola infection, managing Ebola outbreak largely depends on early case detection and surveillance, real-time communication of surveillance data, and Ebola case management. Here we assessed the possibility of uploading data obtained by Internet of Internet device that monitors cellphone company's Call Data Records (CDR), national demographic census, national transportation system and Ebola vaccine production databases on a Blockchain platform to conduct real-time Ebola contact tracing, transmission pattern surveillance and vaccine delivery.
Results: Mobility data obtained by Internet of Things (IoT) from CDR from cellphone companies, national transportation system, and census demographic data can be integrated into a Blockchain platform to provide real-time Ebola surveillance and contact-tracing. While cellphone data provides a unique opportunity to quantify human mobility, Blockchain system magnifies such opportunity by making the data accessible to many actors in real-time. By mounting data from CDR, national population census, national transport system and Ebola vaccine production database on a Blockchain platform will provide additional lens in our understanding of the role played by human population dynamics in the spread as well and containment of Ebola during outbreaks.

Key Words: Internet of things, Blockchain, Ebola, Call data records, Cellphone

\section{LIST of ABBREVIATIONS}

CDR - Call Data Record

IoT - Internet of Things

SMS - Short Messaging Service

\section{INTRODUCTION}

In early August 2018, the Democratic Republic of Congo (DRC) experienced its $10^{\text {th }}$ Ebola outbreak1 since the disease was discovered in 1976. This Ebola outbreak in North Kivu, DRC which came few weeks after that country with the aid of the World Health Organization (WHO) [1] extinguished a similar one posed so much worry for public health officials because it was taking place in a region that is heavily populated, highly trafficked, having many porous international borders, and is the epicenter for an ongoing conflict involving more than 30 different groups of armed militia fighters. Ebola thrives in such fluid and highly mobile human population. In the absence of any approved therapeutics and vaccine to treat or prevent Ebola infection, managing Ebola outbreak largely depends in early case detection and surveillance, real-time communication of surveillance data, and Ebola case management. Here we assessed the possibility of uploading data obtained by Internet of Things devices that monitors cellphone company's CDRs, demographic census, national transportation system and Ebola vaccine production databases on a Blockchain platform to conduct real-time Ebola contact tracing, transmission pattern surveillance and vaccine delivery. We are proposing for the installation of a neatly linked network of smart devices that can automatically process, analyze, and track tagged Ebola surveillance data as they enters the Blockchain platform [2]. Over the past years tremendous efforts have been expended on the use of recent technology to understand the spatial transmission of infectious diseases. Mobility data obtained from cellphone companies has been used to understand the transmission of infectious diseases like influenza [3,4], cholera [5], malaria [6,7], measles [8], dengue [9,10], polio [11] and Ebola [12,13]. The open software online tool HealthMap [14] was the first to flag the West Africa Ebola outbreak in 2014 [15]. Cellphone technology alongside other mash-up digital disease surveillance online tools are changing the speed by which surveillance data for emerging infectious disease are being gathered and reported in real-time.
When a person is confirmed to have Ebola one of the first steps is to trace that person's previous contacts. In big towns and cities with massive transit system and high human trafficking like North Kivu, DRC contact tracing can be time consuming and chaotic. One emerging innovative technology that can also be harnessed to provide real-time surveillance and contact-tracing data in tandem with cellphone technology is Blockchain [16]. First conceptualized by Satoshi Nakamoto in 2008 [16]. Blockchain systems is a cryptographic ledger in digital format that can record strings of transactions from different actors. A Blockchain could be viewed as cryptographic blocks of databases linked together. The cryptographic feature of Blockchain prevents data sharing between untrustworthy or pseudonymous non-outbreak infectious disease surveillance actors to trustworthy players. The past decades have also seen a great stride in the use of cellphone technology for disease surveillance. Every call or text message via Short Messaging Service (SMS) on cellphone is enrouted through the closest cellphone tower of the cellphone subscriber's company. Cellphone company operators lodged the tower code and the Subscriber Identification Module (SIM) card for each caller or SMS sender in their CDRs. If these CDRs and their respective tower maps are combined the call or SMS message origin can be identified. The CDR database of these cellphone companies can be monitored by an Internet of Things (IoT) device mounted on the CDR server.

An IoT is a scientific scenario that provides unique identifiers to objects, animals or people and has the ability to transfer data over a network without human-to-human or human-to-computer interaction (https: internetofthingsagenda.techgate.com). IoT connects devices, systems, and services beyond machine-to-machine communications (M2M) and cover a variety of protocols and domains. For the effective surveillance of Ebola confirmed, probable or suspect cases in chaotic communities such as North Kivu, DRC a Smart Ebola Surveillance IoT System (SERIS) and Smart backbone device are essential. SERIS will serve as the main server/s of the National Ebola Surveillance Unit (NESU) database of the outbreak host country. SERIS will be composed of a Radio Frequency Identification system called SmartIRFID (https: internetofthingsagenda.techgate.com). The function of the SERIS is to create a smart database of tagged Ebola confirmed, probable and suspect cases as well as to disseminate these tagged cases to the Smart backbone device via the internet. Prior to SERIS collating

${ }^{1}$ Center for International Health, University of Munich (LMU), Germany; ${ }^{2}$ Department of Environmental Health Sciences, Njala University, Sierra Leone; ${ }^{3}$ Faculty of Health ARCCIM, University of Technology Sydney, Sydney, Australia; ${ }^{4}$ School of Nursing, Midwifery and Indigenous Health, Faculty of Science, Charles Stuart University, Orange, New South Wales, Australia; ${ }^{5}$ Unit of Information and Communication Technology, Njala University, Sierra Leone; ${ }^{6}$ Department of Nursing, Njala University, Sierra Leone; ${ }^{7}$ Library, Njala University, Sierra Leone; ${ }^{8}$ Massachusetts School of Public Health, University of Massachusetts, Amherst, MA, USA

${ }^{*}$ Correspondence: Jia Bainga Kangbai Center for International Health, University of Munich (LMU), Germany, e-mail Jia.Kangbai@lrz.uni-muenchen.de

Received: August 16, 2018, Accepted: September 11, 2018, Published:September 18, 2018

open Access

This open-access article is distributed under the terms of the Creative Commons Attribution Non-Commercial License (CC BY-NC) (http:// creativecommons.org/licenses/by-nc/4.0/), which permits reuse, distribution and reproduction of the article, provided that the original work is properly cited and the reuse is restricted to noncommercial purposes. For commercial reuse, contact reprints@pulsus.com 
tagged Ebola confirmed, probable and suspect cases, NESU would have known the trend of Ebola in that community, capable of decision making relating to the symptoms of Ebola, have access to a timely and accurate survey on Ebola, capable of giving a timely alert, raising awareness among community members regarding Ebola, evaluate the stock of trial therapies and vaccines for Ebola, as well as preventing the underreporting of Ebola cases. The NESU will have one active SERIS that will collates, analyze and process the Ebola surveillance data obtained from field offices and hospital sources. The task of the SERIS will be to analyze the Ebola surveillance data that have been collected and track its trend within the data entered, analyzed such syndromic data, check whether the collective symptoms can be a sign of other diseases other than Ebola. The main function of the SmartIRFID component of SERIS is to automatically identify tagged confirmed, probable or suspect Ebola cases. The SmartIRFID will thus maintain a confirmed, probable or suspect Ebola case record which will be automatically process and transfer to the Smart IoT Backbone Network (SmartIBN). This SmartIBN will further process, interpret the Ebola surveillance data received from SmartIRFID and mounts on a Blockchain platform. SERIS and SmartIBN devices can also be linked with a cellphone company's tower map and CDR database but in this case they will be monitoring the location of tagged Ebola confirmed, suspected or probable cases based on their calls and SMS messages. SmartIBN on the tower map and CDR database of the subscriber's cellphone company will later transmit the analyzed data of the tagged Ebola confirmed; suspected or probable cases unto a Blockchain platform could be used to track new Ebola cases contacts for Ebola cases. Mounting on a Blockchain platform of tagged surveillance data that have been obtained by SERIS and SmartIBN devices that were embedded in NESU and the CDR database of cellphone company can increase the range by which the many and varied outbreak surveillance agencies can conduct contact tracing during Ebola outbreak management since cellphone and Blockchain have high social scalability. For this surveillance system to work effectively it has to be well coordinated to include public health emergency actors and non-actors such as national transport services, sea port, airport, tourism and commerce. Additionally, this coordination can be made to involve both national and international partners, as well as municipal and state companies in which SmartIRFID and SmartIBN devices can be mounted on their databases in order to provide surveillance data which can be mounted as digital blocks on a Blockchain platform. One typical example of such coordination between a public health agency and non-agency is the incorporation of Orange Telecom into the Data for Development Challenge project (information available at: http://www.d4d.orange.com). With the coordination between public health agency and non-agency in place, the Blockchain platform will provide a secured way of communicating in real-time information about confirmed, probable, and suspected Ebola cases respectively with the different healthcare providers and actors. This concept can be exploited further by the including tagged data obtained from Ebola vaccine and national transportation system databases that have been extracted by embedded SmartIRFID and SmartIBN devices. Ebola vaccine firms can construct a rigid database to include every facet of its production process for each individual serum. For each Ebola trial vaccine dose a specific batch number is provided which if scanned can produce its entire production process information. This batch number can be monitored by a SmartIRFID and the information processed and sent to a SmartIBN device which further analyzed and validate if before mounting it unto the Blockchain platform. This batch number will assuage fears and concerns of both vaccine recipient and healthcare stakeholders who needs to be convinced of its safety. Previous attempts to conduct an Ebola ring vaccination in West Africa suffered setbacks because vaccine recipients were not sure of the safety of the trial vaccine in the first place [17]. A SmartIRFID and SmartIBN devices can also be installed in every facet of the national transportation system to monitor the movement of confirmed, suspect or probable Ebola cases through ticket sales for traveling by airline, railways, and roads as well from the registers at regional and international border posts (Figure 1). The collected data will also be mounted on a Blockchain. The cryptographic feature of Blockchain ensures the safe operation of such database even in the presence of a third party. This system can be accelerated even further if SmartIRFID and SmartIBN devices are mounted on national census databases and the collected demographic census data on residents within the Ebola outbreak zone data is mounted as digital blocks on a Blockchain platform. This will provided added advantage into understanding the influence of demographic factors such as age, occupational and educational status in the transmission of Ebola-again in real-time. Such Blockchain database linkage will shorten the current time it takes for various stakeholders using the Blockchain platform to trace, and track the spread of the outbreak. The current method of sharing information relating to disease outbreak is often long and tortuous and involves government bureaucracy where privacy rules on who can access and edit information. The deficiency

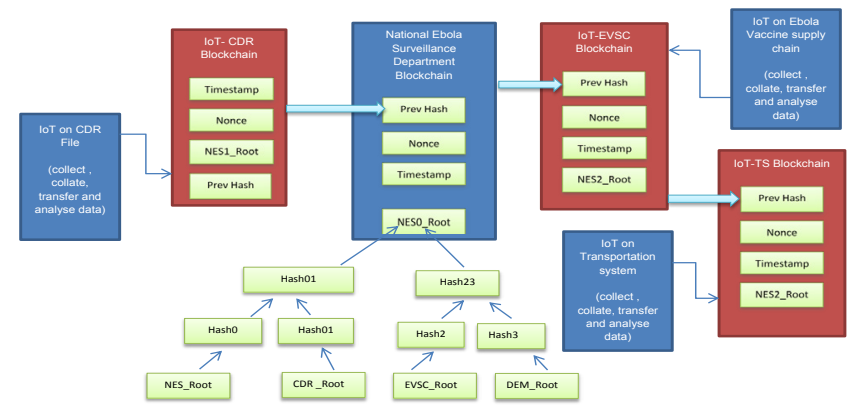

Figure1) A schematic diagram of a Blockchain platform containing data obtained by internet of things devices embedded on cellphone company's call data records $(C D R s)$, databases of national Ebola surveillance department, Ebola vaccine supply chain (EVSC) and national transportation system (TS) are networked

in infectious disease surveillance data alongside its paucity is often blamed for the increase in Ebola cases and mortality rates. This unique advantage that is obtained from mounted CDRs and demographic data via IoT on a Blockchain can be amplified further if the Blockchain network is hosted on a cloud platform.

\section{LIMITATIONS}

There is a growing uncertainty about the future outbreak of Ebola as indicated in the recent outbreak in North Kivu, DRC- hence more work is needed in the area of tracking confirmed, probable and suspected Ebola cases in real-time. Tracking in real-time the human spread of an infectious disease and its transmission pattern never promises to be easy. Our work is prone to many challenges if it is to succeed. Mobile phone data accessibility still remains challenging and restricted by telecommunication laws in many countries. Most times when CDR data are allowed to be used for various purposes it is subjected to further restrictions in terms of duration of usage, quantum of subscribers whose data are to be used, as well as the spatial coverage of the CDR. Additionally even when these CDR data are accessed, mobile phone ownership represents skewed sample of the national population. Most people including children who form the bulk of the atrisk group are underrepresented or totally left out [18]. One study however reported that cellphone accessibility is not affected by ownership [19] while another has demonstrated that mobility estimates are not significantly skewed by cellphone ownership bias [20] in this situation additional work is needed to understand the connection between CDR-derived mobility pattern is linked with normal travel pattern. Our innovative and phenomenological surveillance method can be used simultaneously with other traditional active case finding surveillance methods to validate our concept. While cellphone data provides a unique opportunity to quantify human mobility, Blockchain system magnifies such opportunity by making the data accessible to many actors in real-time. By mounting data from CDR, national population census, national transport system and Ebola vaccine production database on a Blockchain platform will provide additional lens in our understanding of the role played by human population dynamics in the spread as well and containment of Ebola during outbreaks

FUNDING SOURCE: The authors received no funding for this work.

CONFLICT OF INTEREST: The authors declare that they have no competing interests.

\section{REFERENCE}

1. WHO. Cluster of presumptive Ebola cases in North Kivu in the Democratic Republic of the Congo. http://www.who.int/news-room/ detail/01-08-2018-cluster-of-presumptive-ebola-cases-in-north-kivu-inthe-democratic-republic-of-the-congo

2. Report of an International Commission. Ebola haemorrhagic fever in Zaire, 1976. Bull World Health Organ. 1978; 56: 271-93

3. Gog JR, Ballesteros S, Viboud C et al. Spatial Transmission of 2009 Pandemic Influenza in the US. PLoS Comput Biol. 2014; 10: e1003635

4. Ferguson NM, Cummings DA, Cauchemez $S$ et al. Strategies for containing an emerging influenza pandemic in Southeast Asia. Nature. 2005; 437: 209-14

5. Mari L, Bertuzzo E, Righetto L et al. Modelling cholera epidemics: the role of waterways, human mobility and sanitation. J R Soc Interface. 2012; 9:376-88. 
6. Prothero RM. Disease and mobility: a neglected factor in epidemiology. Int J Epidemiol. 1977; 6:259-67.

7. Wesolowski A, Eagle N, Tatem AJ et al. Quantifying the impact of human mobility on malaria. Science. 2012; 338:267-70.

8. Grenfell BT, Bjornstad ON, Kappey J. Travelling waves and spatial hierarchies in measles epidemics. Nature. 2001; 414:716-23.

9. Cummings DA, Irizarry RA, Huang NE et al. Travelling waves in the occurrence of dengue haemorrhagic fever in Thailand. Nature. 2004; $427: 344-7$

10. Stoddard ST, Forshey BM, Morrison AC et al. House-to-house human movement drives dengue virus transmission. Proc Natl Acad Sci. 2013; 110:994-9.

11. Stoddard ST, Morrison AC, Vazquez-Prokopec GM et al. The role of human movement in the transmission of vector-borne pathogens. PLoS Negl Trop Dis. 2009; 3:e481.

12. Wilder-Smith A, Leong WY, Lopez LF et al. Potential for international spread of wild poliovirus via travelers. BMC Med. 2015; 13:133.

13. Poletto C, Gomes MF, Pastore Y, et al. Assessing the impact of travel restrictions on international spread of the 2014 West African Ebola epidemic. Euro Surveill. 2014; 19:20936.
14. Brownstein JS, Freifeld CC. HealthMap: the development of automated real-time internet surveillance for epidemic intelligence. Eurosurveillance. 2007; 12: E0711295.

15. Milinovich G J, Magalhães R J S, Hu W. Role of big data in the early detection of Ebola and other emerging infectious diseases. The Lancet. 2015; Vol 3.

16. Nakamoto S. Bitcoin: A Peer-to-Peer Electronic Cash System. https:// bitcoin.org/bitcoin.pdf

17. http://www.cnn.com/2014/09/19/health/ebola-guinea-killing/index. html.

18. Marshall JM, Toure M, Ouedraogo AL et al. Key traveller groups of relevance to spatial malaria transmission: a survey movement patterns in four sub-Saharan African countries. Malar J. 2016; 15:200.

19. James J, Versteeg M. Mobile phones in Africa: how much do we really know? Social Indicators Research. 2007; 84(1):117-26.

20. Wesolowski A, Eagle N, Noor AM, et al. The impact of biases in mobile phone ownership on estimates of human mobility. J R Soc Interface. 2013; 10(81):20120986 